\title{
Case report \\ A rare presentation of a rare metabolic disorder - Multiple Acyl CoA dehydrogenase deficiency presenting as rhabdomyolysis and renal failure
}

\author{
Arpita Chakraborty ${ }^{1}$, Weena Stanley ${ }^{2}$, M. Mukhyaprana Prabhu ${ }^{3}$ \\ Research Scholar ${ }^{1}$, Associate Professor ${ }^{2}$, Professor ${ }^{3}$, Department of General Medicine, Kasturba Medical College Manipal, \\ Manipal Academy of Higher Education, Manipal-576104, Karnataka, India
}

(Received: July 2021 Revised: October $2021 \quad$ Accepted: November 2021)

Corresponding author: M. Mukhyaprana Prabhu. Email: mm.prabhu@manipal.edu

\begin{abstract}
Multiple acyl-CoA dehydrogenase deficiency (MADD) is a rare metabolic disorder of oxidation of amino acids and fatty acids with an autosomal recessive inheritance. Patients usually present symptoms of MADD in the neonatal period, though it can also be diagnosed in the late adulthood. We present a 36-year-old male with MADD who had sensory axonal neuropathy, rhabdomyolysis metabolic acidosis, lactic acidosis, hypoglycemia and low ketone bodies and renal failure. Early diagnosis and prompt management with carnitine and riboflavin supplements can help in better management of this rare metabolic disorder.
\end{abstract}

Keywords: Multiple acyl-CoA dehydrogenase deficiency; glutaric aciduria type II; rhabdomyolysis; riboflavin; carnitine.

\section{INTRODUCTION}

M ultiple acyl-CoA dehydrogenase deficiency (MADD), formerly known as glutaric aciduria type II, is a very rare metabolic disorder of oxidation of amino acids and fatty acids which manifests since birth. It has an autosomal recessive inheritance (1). Though MADD is usually diagnosed during the childhood or early adulthood, there remains a possibility of detection of this deficiency at an advanced age. The magnitude of MADD in the common population worldwide is only 9 among 100000 and the magnitude of being present since birth is nearly 1 among 200000 (2). Three groups of symptoms usually appear in this disease which are related to the defect in the metabolism of Electron Transfer Flavoprotein (ETF). Patients with type I or II MADD develop symptoms in the neonatal period which includes metabolic acidosis of severe nature, along with hyperammonemia and hyperglycemia. Those having type III MADD, develop common symptoms like weakness in muscles, intolerance to exercise, myalgia and rarely rhabdomyolysis. Infants affected with MADD usually die at an early age but, those who manage to survive are found to suffer from metabolic decompensation occurring repeatedly which resembles Reye's syndrome and gradually leads to the development of hypertrophic cardiomyopathy (3). Individuals who develop the disease at a later age are also found to develop sensory neuropathy along with proximal myopathy. Here, we report a case of 36-year-old male who got admitted to the ICU of a tertiary care hospital with complaints of myalgia in the region of trapezius, hamstrings and paravertebral muscles. He was diagnosed with MADD along with sensory axonal neuropathy, metabolic acidosis, lactic acidosis, hypoglycemia, low ketone bodies, rhabdomyolysis and renal failure. The diagnosis and therapeutic approaches to treat this acute decompensation of MADD is illustrated in this case report.

\section{Case presentation}

A 36-year-old male presented to our institute with symptoms of myalgia in the region of trapezius, hamstrings and paravertebral muscles, pain in the hip joint, which was gradually radiating upwards, chest discomfort since last 3 months and altered sensorium. His muscle pain used to exacerbate during work which initially started involving hamstrings but, later spread to trapezius and paraspinal muscles. Recently, the pain has become more severe which made him to limp. The patient also complained of exertional chest pain, exertional palpitation, breathlessness and vomiting for 4 days. He had recurrent hypoglycemic attacks. There was no history of diabetes. There was no history of fever, giddiness, jaundice, or hypoglycemic attacks. The patient is a reformed smoker and reformed alcoholic. He also had infertility due to low sperm count. There was no history of thyroid dysfunction.

On examination he was found to be hemodynamically stable. He had normal vitals and oxygen saturation level. There was mild pallor but no icterus and lymphadenopathy. Locomotor examination revealed muscle tenderness in bilateral trapezius, paravertebral muscles and hamstrings.

During his admission, Neurological examination revealed proximal muscle weakness and preserved reflexes. No objective evidence of palpitations, altered bowel habits, syncope and loss of consciousness were found. His lab reports showed low TSH, normal T4, but, high free T4. CPK (5994 U/L) and transaminases were also highly elevated (AST/ALT- 2094/940 U/L) with impaired renal parameters (Creatinine- 5.3 $\mathrm{mg} / \mathrm{dl}$ ). Patient was subsequently initiated on renal 
replacement therapy and anti- encephalopathy measures were taken. Antibiotics were thereafter started which subsequently improved his sensorium. LFT levels started showing improvement. Diagnostic work up for evaluation of myopathy and glycogen storage disorders were done. Sensory nerve conduction and motor nerve conduction studies showed sensory axonal neuropathy. Lab reports also revealed metabolic acidosis, lactic acidosis, hypoglycemia and low ketone bodies. USG abdomen and pelvis showed mild coarse echotexture of liver and so autoimmune liver profile was sent which thereafter, came negative. Test for myasthenia gravis also came negative.

The muscle biopsy showed fine vacuoles in many of the fibers which suggested a possible hypokalemic periodic paralysis. The fascicular architecture of the skeletal muscle tissue was maintained. Special stains for lipid (oil red $\mathrm{O}$ ) did not reveal lipid storage myopathy.

Gas chromatography- Mass Spectrometry analysis of organic acids in urine were done which confirmed the presence of suberyl glycine which was found to be consistent with multiple acyl CoA dehydrogenase deficiency (MADD).

Table 1: Gas chromatography- Mass Spectrometry analysis of organic acids in urine

\begin{tabular}{|c|c|}
\hline Retention time & Compound \\
\hline 9.214 & Lactic Acid \\
\hline 14.21 & Urea \\
\hline 17.609 & Internal standard \\
\hline 26.283 & Internal standard \\
\hline 34.654 & 2-oxo-glutaric acid \\
\hline 71.956 & Hippuric acid \\
\hline $\mathbf{5 3 . 3 9 4}$ & Suberyl glycine \\
\hline
\end{tabular}

Colorimetric test was done in the serum of the patient for free fatty acid (FFA) and $\beta$-hydroxybutyrate estimation which showed elevated levels of FFA and $\beta$-hydroxybutyrate. Elevated levels of FFA in presence of hypoglycemia and other clinical features suggested a possibility of fatty acid oxidation defects, whereas low FFA in presence of hypoglycemia was indicative of hyperinsulinemic hypoglycemia.

Table 2: Colorimetric test for free fatty acid (FFA) and $\beta$-hydroxybutyrate estimation in serum

\begin{tabular}{|c|c|c|c|}
\hline \multirow{2}{*}{ Parameters } & \multicolumn{2}{|c|}{ Observed values } & \multirow{2}{*}{ Normal ranges } \\
\cline { 2 - 3 } & Fasting & Post prandial & \\
\hline NEFA & $1.756 \mathrm{mMol} / \mathrm{L}$ & $2.099 \mathrm{mMol} / \mathrm{L}$ & $0.133-0.455 \mathrm{mMol} / \mathrm{L}$ \\
\hline$\beta$-hydroxybutyrate & $1.158 \mathrm{mMol} / \mathrm{L}$ & $1.249 \mathrm{mMol} / \mathrm{L}$ & $0.02-1 \mathrm{mMol} / \mathrm{L}$ \\
\hline FFA/BHB & 1.51 & 1.68 & $0.5-5$ \\
\hline
\end{tabular}

Carnitine/acyl carnitine profile was done which showed normal total carnitine, normal amino acids, low free carnitine, and low free/acyl carnitine ratio. Acyl carnitine profile suggested a possibility of MADD (Glutaric aciduria type 2). MS/MS- Acyl Carnitine profile was also done which suggested elevated C5Dc along with elevated C6, C8 and C10, suggesting a possibility of MADD (Glutaric aciduria type 2).

He was started on thyroxin, empirical antibiotics, muscle relaxants and anti-encephalopathy supplements. He was also started on $\mathrm{L}$ carnitine supplement $(500 \mathrm{mg})$ and riboflavin supplements (100 $\mathrm{mg}$ ). He responded well to the treatment and was thereafter discharged and was asked to continue with the above-mentioned medications.

\section{DISCUSSION}

MADD is a disorder of autosomal recessive nature, which occurs due to the deficiency of ETF dehydrogenase. Impairment in the metabolism of fatty acids along with muscular weakness is presenting feature when diagnosed in late adulthood $(4,5)$. In our patient genetic studies could not be conducted due to poor financial condition of the patient. There are several genetic tests available to confirm the diagnosis of this metabolic disorder such as the ETF and ETFQO encoding gene tests. ETF DH mutation test is considered in patients with MADD who show improvement after treatment with riboflavin. The genes involved are ETF A, ETF B, and the gene for ETF dehydrogenase (6). The magnitude rate of this disease is lower than the other mitochondrial $\beta$ oxidation dysfunction diseases such as medium acyl CoA dehydrogenase deficiency (MACD). Deficiency of riboflavin, elevated levels of urinary glutaric acid, 2-hydroxyglutaric acid, adipic, butyric, lactic, ethylmalonic, and isovaleric acids levels in urine lead to the confirmation in the diagnosis of MADD. Increased short, medium, and long-chain acyl carnitines along with secondary carnitine deficiency are also found in the plasma acyl carnitine profile in MADD patients. Muscle biopsy also confirmed the diagnosis of lipid storage myopathy (2). In a review article by Grunert which involved 350 patients it was found that $85 \%$ of the patients suffering from MADD had myalgia, muscle weakness and intolerance to exercise while $95 \%$ of the patients responded well to riboflavin (5).

In this case nerve conduction studies showed sensory axonal neuropathy. Elevated levels of FFA and $\beta$ hydroxybutyrate were found in gas chromatography/mass spectrometry analysis of organic acids in urine. Muscle biopsy showed possible hypokalemic periodic paralysis. Acyl carnitine profile suggested elevated C5Dc along with elevated C6, C8 and C10, suggesting a possibility of MADD. Carnitine/acyl carnitine profile showed low free 
carnitine and low free/acyl carnitine ratio suggestive of MADD. Gas chromatography/mass spectrometry analysis of organic acids in urine were done which confirmed the presence of suberyl glycine, consistent with MADD. Rhabdomyolysis was also present.

There are only 3 cases of MADD from India. One of them showed the MRI results of a 7-month-old baby presenting with encephalopathy and the other case was MADD with bipolar affective disorder $(7,8)$. The third case was MADD presenting as a lipid storage myopathy (2). This is an extremely rare case report of MADD with multiple conditions such as sensory axonal neuropathy, rhabdomyolysis and encephalopathy. Aggressive treatment with coenzyme $\mathrm{Q}$, carnitine, riboflavin, muscle relaxant and antiencephalopathy measures improved the condition of the patient.

\section{CONCLUSION}

Even though MADD is common in early infancy and childhood, it can also be diagnosed in late adulthood. Early diagnosis and prompt treatment with supplements of riboflavin and carnitine can prevent the complications and help in achieving a better outcome.

\section{ACKNOWLEDGEMENT}

We acknowledge the co-operation of the patient for giving consent to gather the details for this case report.

\section{CONFLICT OF INTEREST}

We state that this case report does not have any conflict of interest.

\section{REFERENCES}

1. Prasun, P. Multiple acyl-CoA dehydrogenase deficiency. GeneReviews®[Internet]. 2020 Jun 18.

2. Chautard, R., Laroche-Raynaud, C., Lia, A. S., Chazelas, P., Derouault, P., Sturtz, F., et al., A case report of a mild form of multiple acyl-CoA dehydrogenase deficiency due to compound heterozygous mutations in the ETFA gene. BMC medical genomics. 2020 Dec; 13(1): 1-6.

3. Ding, M., Liu, R., Qiubo, L., Zhang., Kong, Q. Neonatalonset multiple acyl-CoA dehydrogenase deficiency (MADD) in the ETFDH gene: A case report and a literature review. Medicine. 2020 Sep 11; 99(37).

4. Frerman, F. E. Defects of electron transfer flavoprotein and electron transfer flavoprotein-ubiquinone oxidoreductase Glutaric aciduria type II. The metabolic and molecular basis of inherited disease. 2001: 2357-2365.

5. Grünert, S. C. Clinical and genetical heterogeneity of lateonset multiple acyl-coenzyme A dehydrogenase deficiency. Orphanet journal of rare diseases. 2014 Dec; 9(1): 1-8.

6. Olsen, R. K., Olpin, S. E., Andresen, B. S., Miedzybrodzka, Z. H., Pourfarzam, M., Merinero, B., et al., ETFDH mutations as a major cause of riboflavin-responsive multiple acyl-CoA dehydrogenation deficiency. Brain. 2007 Aug 1; 130(8): 2045-2054.

7. Mumtaz, H. A., Gupta, V., Singh, P., Marwaha, R. K., Khandelwal, N. MR imaging findings of glutaric aciduria type II. Singapore Med J. 2010 Apr 1; 51(4): 69-71.

8. Nanjundappa, G. B., Desai, G., Chaturvedi, S. K. Glutaric acidemia type II associated with bipolar affective disorder. German J Psychiatry. 2011 Jan 1; 14: 48-50. 